\title{
Bedeutung von Zertifizierung und Zentrenbildung für die Viszeralmedizin
}

\author{
Stefan Post ${ }^{\mathrm{a}} \quad$ Michael Betzler $^{\mathrm{b}}$ \\ ${ }^{a}$ Chirurgische Klinik, Universitätsmedizin Mannheim, \\ ${ }^{\mathrm{b}}$ Klinik für Chirurgie I, Alfried Krupp Krankenhaus Rüttenscheid, Essen, Deutschland
}

Kaum ein Begriff wird im deutschen Gesundheitswesen inzwischen so überstrapaziert und inflationär verwendet wie «Zentrum». Dabei lässt dieser Begriff sowohl für den Mediziner/Zuweiser als auch für den Laien/Patienten Assoziationen anklingen an Konzentration, Spezialisierung, besondere Expertise usw. Diese Versprechungen können aber kaum eingelöst werden, solange das Etikett «Zentrum» ungeschützt und selbst verliehen ist.

Es ist gerade einmal 8 Jahre her, als in Nordrhein-Westfalen die Politik und kurz darauf die Fachgesellschaften den Ruf von Betroffenen nach «Brust(krebs)-Zentren» aufgriffen und ein System entwickelten, das in vielerlei Hinsicht beispielgebend wurde. So wurden in unserem Land die Brustzentren die ersten organ- bzw. krankheitsbezogenen Zentren, für die ein stringenter Kriterienkatalog entwickelt wurde, dessen Erfüllung mit einem Zertifikat attestiert werden konnte. Spätestens mit den ersten zertifizierten Darmkrebszentren der Deutschen Krebsgesellschaft (DKG) im Jahr 2006 und den ersten zertifizierten Zentren für Chirurgische Koloproktologie der Deutschen Gesellschaft für Allgemein- und Viszeralchirurgie (DGAV) gemeinsam mit der Deutschen Gesellschaft für Koloproktologie im Jahr 2007 hat diese Welle die Viszeralmedizin erreicht.

Aktuell sind es vor allem diese beiden Fachgesellschaften, DKG und DGAV, die teils ergänzend, teils konkurrierend Zertifikate von viszeralmedizinischer Relevanz vergeben. Das vorliegende Heft versucht, in einem sehr dynamischen, von manchen als inflationär empfundenen Umfeld eine Orientierung zu geben. Im Beitrag von Jost [1] wird das Zertifizierungssystem der DGAV vorgestellt und kommentiert. Schepp und Heitland [2] erläutern die Voraussetzungen und Implikationen eines viszeralmedizinischen Tumorzentrums nach DKG. Kirsch und Herold [3] führen aus, wie aus einem selbst ernannten «Enddarmzentrum» ein zertifiziertes koloproktologisches Exzellenzzentrum wurde. Der Beitrag von Pistorius [4] zeigt an der Entwicklung der eigenen Abteilung, dass die engagierte Beschäftigung mit minimal invasiven Techniken ihre logische Fortsetzung in einer entsprechenden Zertifizierung finden kann. Schließlich liefern Seehofer und Neuhaus [5] eine kritische Übersicht zur Evidenz von Mindestfallmengen und Zentrenbildung in der Leber- und Pankreaschirurgie.

Aus der Summe der Beiträge dürfte klar werden, wie sehr die Trends zur besseren Qualitätssicherung, zur leitliniengesteuerten Behandlung, zu Mindestmengen bei hochkomplexen Prozeduren und zur Ausweisung von zertifizierten Zentren ineinandergreifen. Schon jetzt, nach nur wenigen Jahren der Bildung zertifizierter Zentren, ist absehbar, dass Politik und Kostenträger diese Entwicklungen als Steuerungsinstrument aufgreifen werden (z.B. im Rahmen des Nationalen Krebsplans [6]). Wir alle sollten dafür arbeiten, dass die inhaltliche Ausgestaltung von Zertifizierung und Zentrenbildung weiter in den Händen unserer Fachgesellschaften bleibt.

\section{Literatur}

1 Jost JO: Zertifizierung und Zentrenbildung aus der Sicht der Deutschen Gesellschaft für Allgemeinund Viszeralchirurgie (DGAV). Viszeralmedizin 2011;27:350-358.

2 Schepp W, Heitland W: Bedeutung von Zertifizierung und Zentrenbildung in der Viszeralmedizin. Viszeralmedizin 2011;27:361-366.
3 Kirsch J, Herold A: Erfahrungen mit einem Enddarmzentrum. Viszeralmedizin 2011;27:367-370.

4 Pistorius GA: Erfahrungen in der Entwicklung zum zertifizierten minimal invasiven Zentrum. Viszeralmedizin 2011;27:371-376.
5 Seehofer D, Neuhaus P: Sind Zentren für hepatopankreatiko-biliäre Erkrankungen sinnvoll? Viszeralmedizin 2011;27:377-386.

6 Hohenberger W, Stirkat F, Bruns J, Schmiegel W, Wesselmann S: Krebsregister und Zentrumsbildung. Onkologe 2011;17:135-142.

\section{KARGER \\ Fax +497614520714 \\ Information@Karger.de}

www.karger.com (c) 2011 S. Karger GmbH, Freiburg

$1662-6664 / 11 / 0275-0348 \$ 38.00 / 0$

Accessible online at:

www.karger.com/vim
Prof. Dr. med. Stefan Post

Chirurgische Klinik, Universitätsmedizin Mannheim

Theodor-Kutzer-Ufer 1-3, 68167 Mannheim, Deutschland

Tel. +49 621 383-2225, Fax -3809

stefan.post@umm.de 